\title{
A case: Abscess formation in the sub-Tenon's space after strabismus surgery
}

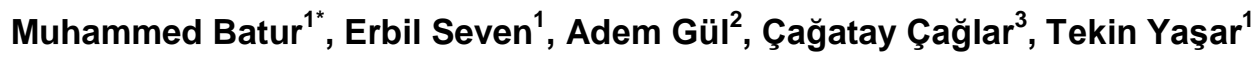 \\ ${ }^{1}$ Department of Ophthalmology, V an Yuzuncu Yil University, Van, Turkey \\ ${ }^{2}$ Department of Ophthalmology, Ondokuz Mays University, Samsun, Turkey \\ ${ }^{3}$ Department of Ophthalmology, Hitit University, Corum, Turkey
}

\begin{abstract}
A five-year-old boy was examined in follow-up in our strabismus department due to exotropia and amblyopia in the right eye. Recession to both lateral recti muscles was performed. When the patient was examined one week, postoperatively, he complained of pain in the right eye. Upon physical examination, hyperemia and minimal swelling of the surgical area were observed. The situation was diagnosed as an abscess. The abscess was drained and the eye was irrigated with cefazolin and gentamicin solutions. All signs and symptoms were determined to be resolved in subsequent postoperative visits.
\end{abstract}

Key Words: Strabismus, Tenon capsule, abscess, surgery

\section{Introduction}

Infection after strabismus surgery is a rare complication that can present with orbital cellulitis, a sub-Tenon's abscess, an infected epithelial inclusion cyst, uveal effusion syndrome, endophthalmitis, or keratitis (1-6). We diagnosed and treated a 5 year-old boy with a sub-Tenon's abscess after strabismus surgery.

\section{Case report}

A five-year-old boy was examined in follow-up in our strabismus department due to exotropia and amblyopia in the right eye. Visual acuity was 20/25 for the right eye and 20/20 for the left eye. Eye movements were normal in all gazes. The patient did not exhibit any systemic disease.

Surgery was conducted under general anesthesia. Eyelids and surrounding area were cleaned with povidone iodine $10 \%$ for three minutes. Povidone iodine $5 \%$ was instilled for three minutes into both fornices before surgery. A seven $\mathrm{mm}$ recession to both lateral recti muscles was performed using a fornix-based approach. Recti were fixated to sclera with $6 / 0$ absorbable sutures (Biosorb, Alcon, USA). Conjunctival incisions were closed with 8/0 absorbable sutures (Biosorb, Alcon, USA). A topical antibiotic (ofloxacin 0.3\%) and a topical steroid (dexamethasone $0.1 \%$ ), each to be administered six times per day, were prescribed as postoperative treatment. The patient was examined and discharged on the first day, postoperatively.

When the patient was examined one week, postoperatively, he complained of pain in the right eye. Upon physical examination, hyperemia and minimal swelling of the surgical area were observed. The sutures at the conjunctival incision site were intact and no reaction in the anterior chamber was noted. The patient was ortophoric in the primary gaze and no limitation or restriction of eye movements was observed. The situation was diagnosed as an abscess. Systemic antibiotic (cefazolin and gentamicin) treatment was initiated and topical antibiotic treatment was increased to one instillation per hour. An abscess in the subTenon's space fistulizing to the conjunctiva was observed in the tenth day, postoperatively (Figure 1). Surgery was performed on the patient under general anesthesia. The conjunctiva was explored, and an abscess was observed in the sub-Tenon's space at the fixation point of the lateral rectus muscle to the sclera. No muscle or scleral involvement existed. The abscess was drained and the eye was irrigated with cefazolin $(500 \mathrm{mg} / 2 \mathrm{cc})$ and gentamicin $(40 \mathrm{mg} / 2 \mathrm{cc})$ solutions.

A culture was sampled from the purulent material. Upon microscopic assessment, the presence of abundant polymorphonuclear leucocytes and

*Corresponding Author: Muhammed Batur MD. Department of Ophthalmology, Yuzuncu Yil University, 65300, Van, Turkey, Phone Number: +90 (532) 56479 56, Fax Number: +90 (432) 21683 52, E-mail address: muhammedbatur@gmail.com 
Gram positive cocci was reported. The results of the culture indicated the presence of Staphylococcus aureus.

All signs and symptoms were determined to be resolved in subsequent postoperative visits. The patient was ortophoric and eye movements were found to be within normal limits for all gazes. Visual acuity was 20/25 in the right eye and 20/20 in the left eye in the sixth month, postoperatively.

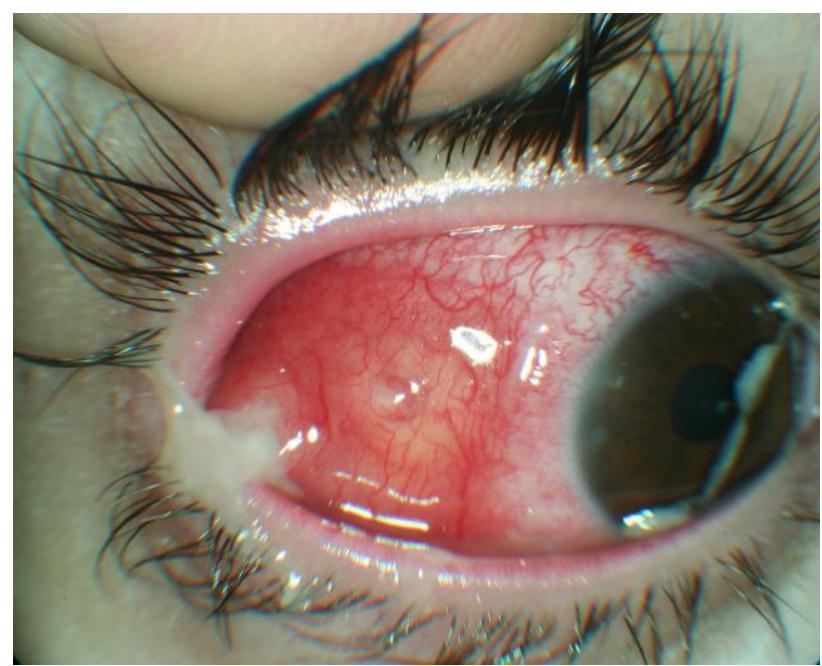

Fig. 1. Fistulizing abscess observed in the tenth day, postoperatively.

\section{Discussion}

Sub-Tenon's abscess is a rare complication after strabismus surgery. In the literature, two reports exist in which abscess formation in the subTenon's space was identified after strabismus surgery.

The first case was reported by Brenner et al. (1) in which a two-year-old boy was diagnosed with accomodative esotropia and a right medial recession was performed. On the third day, postoperatively, fever, conjunctival eryhtema, pain, and lid edema developed. The patient was operated on a second time, and the abscess in the sub-Tenon's space was drained. Culture results revealed the presence of $S$. aureus microorganisms.

A second case was reported by Kothari and Sukri (2) in which a five-year-old girl was diagnosed with esotropia. A surgical procedure was performed involving recession of the medial recti and myectomy to the inferior oblique muscles. The patient was lethargic on the third day, postoperatively, and a mucopurulant discharge from both eyes was observed. Whereas on the first day, postoperatively, the eyes were ortophoric, after a few days, a 30 degree exodeviation and restriction in adduction in the left eye, and a minimal restriction in adduction in the right eye, had developed. When conjunctival exploration was performed, sub-Tenon's abscesses were observed in both areas of the medial recti at which the operation occurred. Microbiologic assessment revealed the presence of $S$. aureus.

In a related study in which periocular infections were observed after strabismus surgery, the most common organisms found were Staphylococcus aureus, Staphylococcus epidermidis, Streptococcus pneumoniae and Haemophilus influenza (7). However, in the aforementioned two cases, (1-2) and in this report, only $S$. aureus organisms were isolated.

In our study, a histopathologic examination was conducted. Highly colonised bacterial involvement, acute inflammation, and necrosis were determined in the pathologic assessment. However, no epithelial cell involvement was observed, which contradicts the argument of Khan et al. (4) who reported that these were not true abscesses, but were infected epithelial inclusion cysts mimicking subconjunctival abscesses.

Kivlin et al. (7) suggested that unsuspected sinusitis, eye rubbing, and poor hygiene were predisposing factors. When the patient was reexamined and re-interviewed, none of these predisposing factors were identified.

Surgeries for strabismus have been conducted in our clinic for 17 years. This was the first case of periocular infection following strabismus surgery that was ever encountered in our practice. No severe complications were observed. Drainage of the abscess must be performed to prevent more severe future complications, such as orbital cellulitis and myositis.

\section{Acknowledgements: None}

Declaration of Interest: The authors report no conflicts of interest. The authors alone are responsible for the content and writing of the paper.

\section{References}

1. Brenner C, Ashwin M, Smith D, Blaser S. SubTenon's space abscess after strabismus surgery. J AAPOS 2009; 13: 198-199.

2. Kothari M, Sukri N. Bilateral Staphylococcus aureus sub-Tenon's abscess following strabismus surgery in a child. J AAPOS 2010; 14: 193-195.

3. Narayanappa S, Dayananda S, Dakshayini M, Gangasagara SB, Prabhakaran VC. Conjunctival inclusion cysts following small incision cataract 
surgery. Indian J Ophthalmol 2010; 58: 423-425.

4. Khan AO, Al-Katan H, Al-Baharna I, Al Wadani F. Infected epithelial inclusion cyst mimicking subconjunctival abscess after strabismus surgery. J AAPOS 2007; 11: 303-304.

5. Recchia FM, Baumal CR, Sivalingam A, et al. Endophthalmitis after pediatric strabismus surgery. Arch Ophthalmol 2000; 118: 939-944.
6. Bedrossian EH. Hypopyon keratitis: Following muscle surgery. Am J Ophthalmol 1966; 61: 15301532.

7. Kivlin JD, Wilson ME. Periocular infection after strabismus surgery. The Periocular Infection Study Group. J Pediatr Ophthalmol Strabismus 1995; 32: 42-49. 